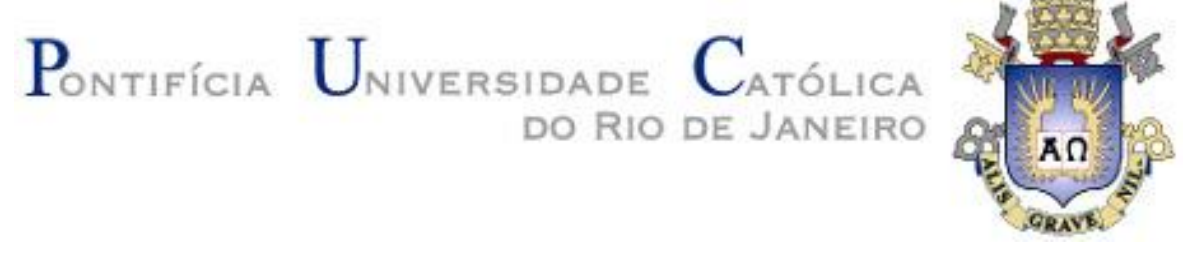

Fábio Francisco de Araujo

Percepções e Significados para o Lazer do Jovem Consumidor de Baixa Renda: Um Estudo Exploratório em uma Comunidade da Zona Sul Carioca

Dissertação apresentada ao Programa de PósGraduação em Administração de Empresas da PUC-Rio como requisito parcial para obtenção do título de Mestre em Administração de Empresas.

Orientadora: Angela Maria Cavalcanti da Rocha Co- Orientadora: Marie Agnes Chauvel

Rio de Janeiro, Maio de 2011 
Fábio Francisco de Araujo

\section{Percepções e Significados para o Lazer do Jovem Consumidor de Baixa Renda: Um Estudo Exploratório em uma Comunidade da Zona Sul Carioca}

Dissertação apresentada como requisito parcial para obtenção do grau de Mestre pelo Programa de Pósgraduação em Administração de Empresas da PUC-Rio. Aprovada pela Comissão Examinadora abaixo assinada.

Profạ. Angela Maria Cavalcanti da Rocha

Orientadora

Departamento de Administração - PUC-Rio

Prof‥ Marie Agnes Chauvel

Co-orientadora

Universidade Federal de São João Del-Rei

Prof. Paulo César de Mendonça Motta Departamento de Administração - PUC-Rio

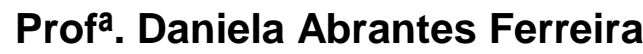
Universidade Federal do Rio de Janeiro

Profa. Mônica Herz Vice-Decana de Pós-Graduação do CCS

Rio de Janeiro, 03 de maio de 2011 
Todos os direitos reservados. É proibida a reprodução total ou parcial do trabalho sem autorização da universidade, do autor e da orientadora.

Fábio Francisco de Araujo

Graduou-se em Administração pela Pontifícia Universidade Católica do Rio de Janeiro (PUC-Rio) em 2006. Graduou-se em Direito pela Universidade Federal do Rio de Janeiro (UFRJ) em 2008. Publicou um trabalho no Congresso Internacional do Instituto Franco-Brasileiro de Administração de Empresas (IFBAE, edição de 2007) e três trabalhos no Encontro Nacional de Pós-Graduação em Administração (Encontros da ANPAD, edições de 2007, 2008 e 2010). Desenvolve pesquisas acadêmicas em Administração focadas em temas das áreas de marketing e de estratégia.

Araujo, Fábio Francisco de

Percepções e significados para o lazer do jovem consumidor de baixa renda : um estudo exploratório em uma comunidade da Zona Sul carioca / Fábio Francisco de Araujo ; orientadora: Angela Maria Cavalcanti da Rocha ; co-orientadora: Marie Agnes Chauvel. - 2011.

129 f. : il. (color.) ; $30 \mathrm{~cm}$

Dissertação (mestrado) - Pontifícia Universidade Católica do Rio de Janeiro, Departamento de Administração, 2011.

Inclui bibliografia

1. Administração - Teses. 2. Comportamento do consumidor. 3. Consumidores de baixa renda. 4. Base da pirâmide. 5. Lazer. I. Rocha, Angela Maria Cavalcanti da. II. Chauvel, Marie Agnes. III. Pontifícia Universidade Católica do Rio de Janeiro. Departamento de Administração. IV. Título. 
Para minha amada mãe, Maria F. Jales de Araujo 


\section{Agradecimentos}

Primeiramente, ao Senhor Deus por minha vida e tudo de bom que eu recebi ou me ajudou a conquistar.

À minha querida mãe, Maria Jales, por todo amor, carinho e apoio dedicado durante toda a minha vida, razão pela qual a considero o grande amor e pessoa mais importante dessa fase de minha vida, para quem dedico este trabalho.

À Profa. Marie Chauvel pelo apoio e incentivo às atividades de pesquisa, por toda ajuda recebida em forma de co-orientação para elaboração desta dissertação, por também poder contar com a sua presença na banca examinadora e, sobretudo, pela fé que creditou em meu potencial enquanto estudante e pesquisador.

À Profa. Angela da Rocha que me orientou nessa dissertação com carinho e interesse, além de seu entusiasmo pelo tema pesquisado neste trabalho.

Ao Prof. Paulo César Motta e à Profa. Maria Angela Campelo de Mello pelas dicas e por participarem da banca examinadora. À Profa. Daniela Abrantes Ferreira por participar da banca de aprovação desta dissertação como examinadora externa. Ao Prof. Jorge Ferreira, coordenador do programa de pós-graduação em Administração, por ajudar na elaboração da análise estatística desta pesquisa.

À aluna de iniciação científica do departamento de Administração da PUC-Rio Marianne Schulze que participou da pesquisa, trazendo importantes contribuições tanto na primeira etapa da pesquisa (entrevistas) quanto na segunda etapa da pesquisa (aplicação dos questionários).

A todos os participantes que contribuíram em ambas as etapas desta pesquisa.

Aos professores que me passaram por minha vida e contribuíram para minha formação, bem como aos amigos mais próximos e companheiros de estudo que estiveram mais presentes e compartilharam alguns passos de minha trajetória. 


\section{Resumo}

Araujo, Fábio Francisco de; Rocha, Angela Maria Cavalcanti da (Orientadora); Chauvel, Marie Agnes (Co-orientadora). Percepções e Significados para o Lazer do Jovem Consumidor de Baixa Renda: Um Estudo Exploratório em uma Comunidade da Zona Sul Carioca. Rio de Janeiro, 2011. 129 p. Dissertação de Mestrado - Departamento de Administração, Pontifícia Universidade Católica do Rio de Janeiro.

A população de baixa renda representa atualmente mais da metade da população brasileira, proporção tal que representa mais de 100 milhões de consumidores em todo país. Esses consumidores vêm aumentando seu potencial de consumo ao longo dos anos e começaram a atrair o interesse de pequenas e grandes empresas a partir de meados da década de 1990. Prahalad (2005) destaca a relevância desse vasto segmento de consumidores e também a necessidade de abordá-los com uma visão inovadora. Dessa forma, as empresas devem aproveitar as oportunidades advindas de um posicionamento estratégico adequado para um enorme número de consumidores de baixa renda, ávidos por adquirir produtos de qualidade e com preços compatíveis com seu poder aquisitivo. Todavia, no Brasil, ainda se sabe muito pouco sobre esses consumidores, que foram, por décadas, praticamente ignorados pelas pesquisas acadêmicas e de mercado. Em especial, o tema do lazer para esses grupos foi muito pouco estudado e, até recentemente, as possibilidades de acesso ao lazer por parte da população de baixa renda no Brasil eram restritas, seja por falta de espaço ou por falta de recursos. Este estudo, de caráter exploratório, investigou o comportamento desse grupo de consumidores no que diz respeito ao lazer: as opções a que têm acesso, suas preferências, as razões de suas escolhas, assim como o significado das atividades de lazer. Para isso, selecionou-se uma comunidade de baixa renda da região sul da cidade do Rio de Janeiro. As entrevistas foram divididas em duas partes, primeiramente foram realizadas entrevistas semi-estruturadas com os líderes da comunidade. $\mathrm{Na}$ segunda etapa, optou-se por utilizar um questionário, aplicados a jovens e adolescentes entre 15 e 25 anos da comunidade. A amostra contou com um total de 86 (oitenta e seis) respondentes. As informações obtidas na segunda etapa da pesquisa foram analisadas, buscando-se compreender e descrever o nível de 
importância concedido pelas jovens e adolescentes ao lazer e analisar as percepções desse grupo de consumidores em relação a sua prática. Verificou-se que esses consumidores entendem e reconhecem o lazer como algo importante para suas vidas. De acordo com os resultados encontrados nesta pesquisa, o lazer se relaciona com questões muito importantes para o bem-estar social das pessoas, tais como qualidade de vida, incentivo à atividade física e valorização da cultura, mas não é reconhecido necessariamente como um produto a ser consumido.

\section{Palavras-chave}

Comportamento do Consumidor; Consumidores de Baixa Renda; Base da Pirâmide; Lazer. 


\section{Abstract}

Araujo, Fábio Francisco de; Rocha, Angela Maria Cavalcanti da (Advisor); Chauvel, Marie Agnes (Co-Advisor). Perceptions and Meanings of Leisure of Low-Income Young Consumers: An Exploratory Study of a Community in the South Zone of Rio de Janeiro. Rio de Janeiro, 2011. 129 p. MSc. Dissertation. Departamento de Administração, Pontifícia Universidade Católica do Rio de Janeiro.

The low-income population represent over than a half of the population, a proportion that represents more than 100 million consumers around the country. These consumers, who are increasing their potential consumption over the years, began to attract the attention of smalls and larges companies from the mid-1990s. Prahalad (2005) highlights the relevance of this large segment of consumers and the necessity to approach them with an innovative vision. In this way, companies must take opportunities resulting from suitable strategic positions for a huge number of low-income consumers eager to buy products with good quality and compatible prices with their purchasing power. In particular, the theme of leisure for these groups has been little studied. In general, until recently, the access possibility of leisure by the low income population in Brazil was restricted by lack of space and resources. This study, with an exploratory character, investigated the behavior of this group of consumers related to the leisure theme: the choices that they have access, their preferences, the reasons for their choices, as well as the meaning of leisure activities. For this, there was a selection of a low income community in the south zone in the city of Rio de Janeiro. The interviews were divided in two parts, first carried out semi-structured interviews with community leaders. In the second step, was chose a semi-structured questionnaire for youths and teenagers of the community between 15 to 25 . The sample had a total of 86 (eighty six) respondents. The information obtained in the second stage of the study were analyzed, seeking to understand and describe the level of importance given by the youth and teenagers to leisure and analyze the perceptions of this group of consumers in relation to the practice of leisure. It was found that these consumers understand and recognize the leisure as something important in their lives. According to the findings of this study, leisure approaches itself from very 
important questions for the social welfare, such as quality of life, incentives to practice physical activities, and culture appreciation, but is not necessarily recognized as a product to be consumed.

\section{Keywords}

Consumer Behavior; Low-income Consumers; Bottom of the Pyramid; Leisure. 


\section{Sumário}

1. Introdução 16

$\begin{array}{ll}\text { 1.1. Objetivo final } & 18\end{array}$

1.2. Objetivos intermediários 18

$\begin{array}{ll}\text { 1.3. Delimitação do estudo } & 18\end{array}$

$\begin{array}{ll}\text { 1.4. Relevância do estudo } & 19\end{array}$

2. Revisão de Literatura 20

2.1. Posição social e consumo 20

2.2. Perfil dos consumidores de baixa renda no mercado brasileiro 25

2.3. Comportamento do consumidor de baixa renda 29

2.4. Estratégias de marketing para a população de baixa renda 37

2.5. O consumidor de baixa renda e o lazer 41

3. Metodologia 48

3.1. Tipo de pesquisa 48

3.2. Seleção dos informantes 51

3.3. Coleta de dados 51

3.4. Análise dos dados 52

3.5. Limitações dos métodos 54

4. Resultados 57

4.1. A comunidade 57

4.2. Perfil dos jovens entrevistados 62

4.3. O lazer para os Jovens de Vila Canoas 68

4.4. Opções de lazer em Vila Canoas 72

4.5. Percepções e significados do lazer 75

$\begin{array}{lll}\text { 4.6. } & \text { Resultados da análise de clusters } & 87\end{array}$

$\begin{array}{ll}\text { 5. Considerações finais } & 101\end{array}$ 
$\begin{array}{ll}\text { 6. Referências bibliográficas } & 106\end{array}$

$\begin{array}{ll}\text { 7. Anexos } & 114\end{array}$

ANEXO I - Roteiro de entrevista utilizado com a representante da Comunidade e presidente da Associação de Moradores de Vila Canoas

ANEXO II - Roteiro de entrevista utilizado com uma integrante da Associação de Mulheres de Vila Canoas

ANEXO III - Roteiro de entrevista utilizado com os jovens Moradores de Vila canoas, aplicado na forma de questionário 


\section{Lista de fotografias}

Fotografia 1 - Mosaico de boas-vindas na entrada da comunidade 58

Fotografia 2 - A comunidade Vila Canoas $\quad 59$

Fotografia 3 - Praça principal da comunidade e visão da sede da AMAVICA 59

Fotografia 4 - Travessas de Vila Canoas 60

Fotografia 5 - Moradias de Vila Canoas $\quad 60$

Fotografia 6 - Capela Católica Nossa Senhora das Graças e Biblioteca de Vila

$\begin{array}{ll}\text { Canoas } & 60\end{array}$

Fotografia 7 - Imagens da ONG PARA TI: Sede, Sala de Reforço Escolar,

Sala de Informática

Fotografia 8 - Jovens iniciando uma "pelada" de futebol no espaço esportivo da ONG PARA TI 


\section{Lista de tabelas}

Tabela 1 - Renda Familiar Mensal - ABEP/2010 31

Tabela 2 - Sexo do público entrevistado $\quad 62$

Tabela 3 - Faixa etária $\quad 63$

Tabela 4 - Nível de instrução $\quad 63$

Tabela 5 - Jovens que declararam exercer atividade remunerada 64

Tabela 6 - Profissão exercida 64

Tabela 7 - Renda Média Mensal Familiar $\quad 65$

Tabela 8 - Número de integrantes da família com renda 65

$\begin{array}{ll}\text { Tabela } 9 \text { - Atividades Praticadas/Mulheres } & 69\end{array}$

Tabela 10 - Atividades Praticadas/Homens $\quad 71$

Tabela 11 - Códigos e número de respondentes sobre os projetos de vida 75

Tabela 12 - Respostas selecionadas sobre projeto de vida para o futuro 77

Tabela 13 - Códigos e número de respondentes sobre o maior sonho 78

Tabela 14 - Respostas selecionadas sobre o maior sonho dos jovens da $\begin{array}{ll}\text { comunidade } & 79\end{array}$

Tabela 15 - Respostas selecionadas sobre a importância de reservar tempo para o $\begin{array}{ll}\text { lazer } & 80\end{array}$

Tabela 16 - Códigos e número de respondentes sobre o tempo livre 81

Tabela 17 - Respostas selecionadas sobre o significado de tempo livre 82

Tabela 18 - Códigos e número de respondentes sobre o lazer $\quad 84$

Tabela 19 - Respostas selecionadas sobre o significado de lazer 86

$\begin{array}{ll}\text { Tabela } 20 \text { - Número de casos em cada cluster } & 87\end{array}$

Tabela 21 - Análise de Variância Multivariada (MANOVA) 88

Tabela 22 - Análise de Variância (ANOVA) $\quad 89$

Tabela 23 - Análise K-means: Iteração Histórica dos Clusters 96

Tabela 24 - Distribuição final dos clusters em relação às variáveis de análise 97 


\section{Lista de gráficos}

Gráfico 1 - Disponibilidade de dinheiro para gastos com lazer (mulheres) 66

Gráfico 2 - Disponibilidade de dinheiro para gastos com lazer (homens) 66

Gráfico 3 - Maior peso nas despesas do orçamento 67

Gráfico 4 - Opções de lazer mais desejadas $\quad 73$

$\begin{array}{ll}\text { Gráfico } 5 \text { - Produtos mais desejados } & 74\end{array}$

Gráfico 6 - Diagrama considerando a média de gênero 90

Gráfico 7 - Diagrama considerando a idade dos respondentes 91

Gráfico 8 - Diagrama considerando se os respondentes moram sozinho 92

Gráfico 9 - Diagrama considerando o nível de instrução 93

Gráfico 10 - Diagrama considerando a colaboração na renda familiar 94

Gráfico 11 - Diagrama considerando a colaboração na renda familiar 95 
Nunca duvide que um pequeno grupo de cidadãos preocupados e compromissados possa modificar o mundo. Na realidade, esta é a única coisa que de fato o tem modificado.

Margaret Mead. 